\title{
La digitalización como eje de transformación de las agencias de medios españolas
}

\author{
Ramón Francisco MARTÍN GUART ${ }^{1}$ \\ José FERNÁNDEZ CAVIA ${ }^{2}$ \\ Departamento de Comunicación \\ Universidad Pompeu Fabra
}

\begin{abstract}
RESUMEN:
El ecosistema de los medios está cambiando debido no solo a motivos coyunturales sino también estructurales entre los que destaca la revolución tecnológica y la consolidación de internet como medio de comunicación. Las agencias de medios deben reformular su modelo de negocio e incorporar, por ejemplo, métricas como el $i G R P$ que permiten justificar las inversiones online con los mismos argumentos que se utilizan con el resto de medios.
\end{abstract}

PALABRAS CLAVE: Agencias de medios; digitalización; planificación de medios; publicidad; internet

TITLE: Digitalization as the axis of transforming the Spanish media agencies.

\section{ABSTRACT:}

The media environment is changing owing to circumstantial and structural reasons, highlighted among them the technological revolution and the consolidation of internet as a media of communication. Media agencies should rethink their business model and incorporate some new metrics, for example, the iGRP that justify online investments with the same arguments used in other media.

KEY WORDS: Media agencies; digitalization; media planning; advertising; internet.

\section{Introducción}

La industria publicitaria vive unos momentos de cambio intenso, de profunda transformación debida no solo a motivos coyunturales, como la recesión económica, sino a motivos estructurales como la globalización de los mercados, la evolución tecnológica o la creación de un nuevo sistema de medios de comunicación que trasciende la mera incorporación de un nuevo medio como es internet.

1 Profesor de Planificación de Medios en la Universidad Pompeu Fabra (Barcelona). Miembro del Grupo de Investigación «Communication, Advertising \& Society» (CAS) perteneciente al Departamento de Comunicación de la UPF. Director de medios en la agencia Mindshare y, anteriormente, en Initiative Media y Media Planning. E-mail: ramon.martin@upf.edu.

2 Profesor titular de Publicidad y Relaciones Públicas en la Universidad Pompeu Fabra (Barcelona). Subdirector de Investigación y Postgrado del Departamento de Comunicación. Director del Grupo de Investigación CAS. Ha publicado diversos libros sobre comunicación y artículos en revistas nacionales e internacionales. E-mail: jose.fernandez@upf.edu. 
El ecosistema mediático ${ }^{3}$ se ha modificado de manera profunda en los últimos años, y nunca volverá a ser el mismo. Según Cardoso, «la comunicación en red está sustituyendo -de forma paulatina, aunque constante- a la comunicación de masas y a sus paradigmas comunicacionales en nuestras sociedades ${ }^{4}$. La publicidad se encuentra todavía en proceso de adaptación a esa nueva realidad ${ }^{5}$. El sistema tradicional de la comunicación de masas, que ha sido el modelo que ha creado y sostenido al negocio publicitario durante más de ciento cincuenta años, está dejando paso a un nuevo paradigma, aún por concretar, pero que implica o bien la reinvención de la publicidad o bien su desaparición tal como la conocemos ${ }^{67} \mathrm{y}$, como prueba de esta tendencia ya en Estados Unidos «un hogar típico tiene más de 26 dispositivos de comunicación, y los consumidores pasan más tiempo usándolos (televisores, radios, ordenadores, iPods, teléfonos móviles, etc.) que en cualquier otra actividad» ${ }^{8}$. Las agencias de publicidad y las agencias de medios se encuentran en proceso de adaptación y deben encontrar su modelo de negocio. Así lo constata Patricia Corredor, investigadora de la Universidad Rey Juan Carlos de Madrid, al afirmar que «el resultado es una reordenación de las estructuras, nuevos organigramas y nuevos perfiles profesionales, que se presentan bajo una filosofía integradora de las estrategias off line y online»».

$\mathrm{Al}$ analizar la inversión publicitaria española, uno de los datos más significativos es el del porcentaje que representa respecto del PIB. Las cifras señalan que hasta el año 2007 se produjo un crecimiento real -en el que la inversión publicitaria creció en porcentaje por encima de la inflación-, sin embargo a partir de ese momento, el índice se ha ido reduciendo notablemente como consecuencia de la disminución en la inversión publicitaria, tanto en medios convencionales como en medios no convencionales ${ }^{10}$. Según puede apreciarse en la tabla 1, en 2011 el porcentaje que representa la inversión publicitaria sobre el conjunto del PIB español se redujo en nueve centésimas, situándose en un escenario de severa contracción de los presupuestos publicitarios $\mathrm{y}$, por tanto, de dificultad financiera para las empresas del sector.

3 BoIX, A. (2007): «Transformaciones en el ecosistema mediático y nuevas pautas de regulación administrativa del hecho audiovisual», Quaderns del CAC, 27, 37-51.

4 CARDoso, G. (2011): «Más allá de internet y de los medios de comunicación de masas», Telos, 86, 1-10.

5 FERNÁNDEZ CAVIA, J. (2005): «Los formatos no convencionales y la transformación del negocio publicitario en la televisión», Quaderns del CAC, 22, 35-48.

6 Vollmer, C., \& Precourt, G. (2008): Always on. Advertising, marketing and media in an era of consumer control, New York, McGraw Hill.

7 Hull, J. (2009): «Conclusion: the future of advertising and advertising agencies» en H. Powell, H. [et al.] (ed), The advertising handbook, London, Routledge.

8 HiLl, R.P. y Moran, N. (2011): «Social marketing meets interactive media: lessons for the advertising community», International Journal of Advertising, 30, 5.

9 CORREDOR LANAS, P. (2011): «Nuevos profesionales en publicidad», Telos, 87, 1-4.

10 Bajo la denominación genérica de «medios no convencionales» se recogen el conjunto de medios publicitarios que, habitualmente, aparecen asimismo enmarcados bajo la expresión inglesa «below the line», como por ejemplo el marketing directo, el marketing móvil, PLV, ferias y congresos, entre otros. 
Fig. 1: Evolución del porcentaje de inversión publicitaria sobre el PIB en España.

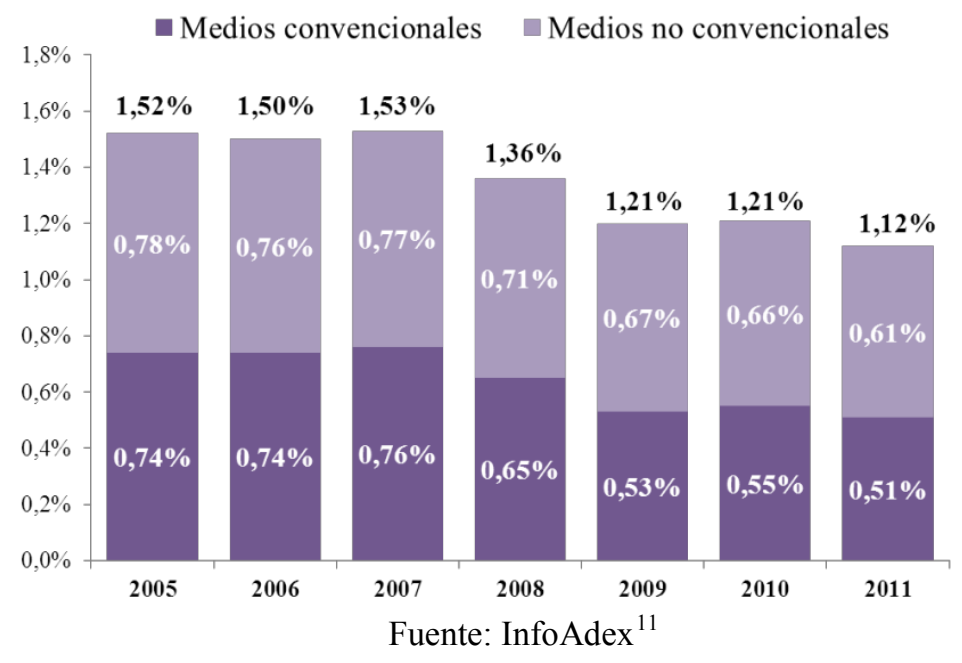

\section{Contexto de la actividad publicitaria: distribución de la inversión por tipo- logía de medios}

Tras el apartado anterior, puede intuirse que en los últimos tiempos la crisis ha sido un referente inevitable a la hora de hablar de inversión publicitaria. Hasta el año 1990, el incremento en las cifras de inversión era cada año de un 24\% como media, pero en 1993 se produce una importante fractura al caer en picado un $31 \%{ }^{12}$. Desde entonces el avance ha sido lento hasta llegar al 2008, año en el que las inversiones publicitarias volvieron a desplomarse.

Partiendo de los datos que publica anualmente Infoadex, que es la principal fuente de información multimedia sobre inversiones publicitarias, vamos a tratar de identificar cambios significativos en la cuota de inversión o share of investment en cada uno de los medios publicitarios en los últimos tiempos. En 2011 la inversión real estimada del mercado publicitario vuelve a contraerse como ocurrió en los años 2008 y 2009, quedando el año 2010 como excepción en el período de estos cuatro años con caídas en sus cifras publicitarias: la inversión alcanzó en 2011 los 12.061 millones de euros, lo que representó un decrecimiento del 6.5\% sobre los 12.893 millones de euros registrados en 2010, año que supuso un crecimiento del $1.4 \%$ respecto al 2009. Esta aparente estabilidad en 2010 oculta, sin embargo, una

11 InfoAdex controla las inversiones publicitarias por medios y soportes, como también la ocupación y las inserciones publicitarias realizadas en todos los medios convencionales: cine, diarios, dominicales, exterior, internet, radio, revistas y televisión.

12 Castellblanque, M. (2001): Estructura de la actividad publicitaria. La industria de la publicidad de la A a la Z. España: Un caso extrapolable, Barcelona, Ediciones Paidós. 
conducta muy dispar entre los diferentes medios ya que mientras la inversión en los medios convencionales creció un $4.0 \%$ respecto al periodo anterior, la inversión en los medios no convencionales todavía no dejó atrás los números rojos al caer el $0.6 \%$ respecto al 2009 , tal y como muestran las dos siguientes tablas.

Fig. 2: Evolución de la inversión publicitaria (en millones de euros).

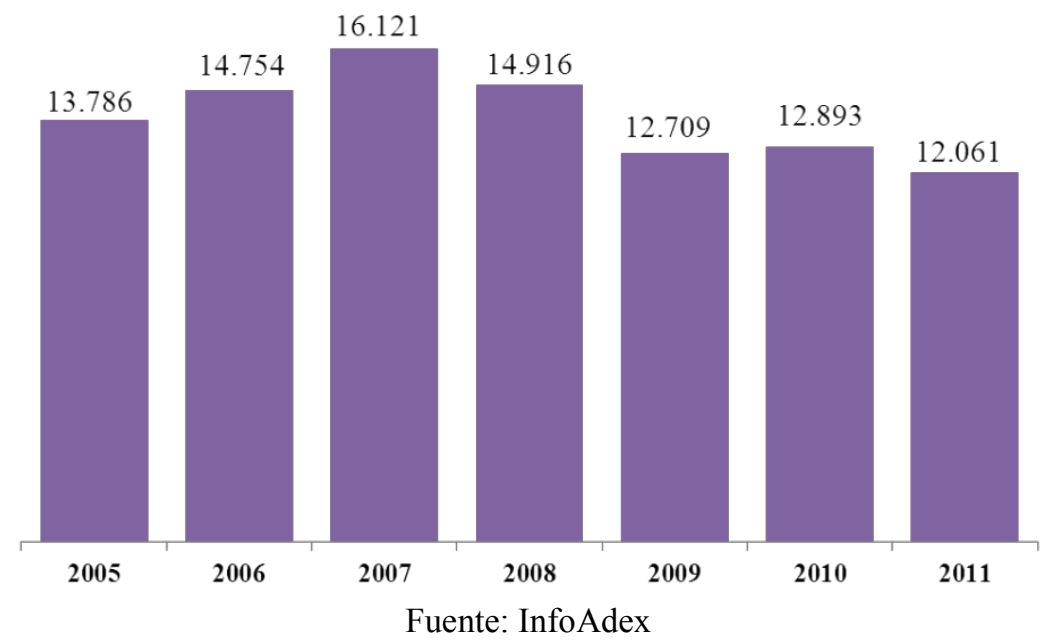

Fig. 3: Evolución de la inversión publicitaria por tipo de medios (en millones de euros).

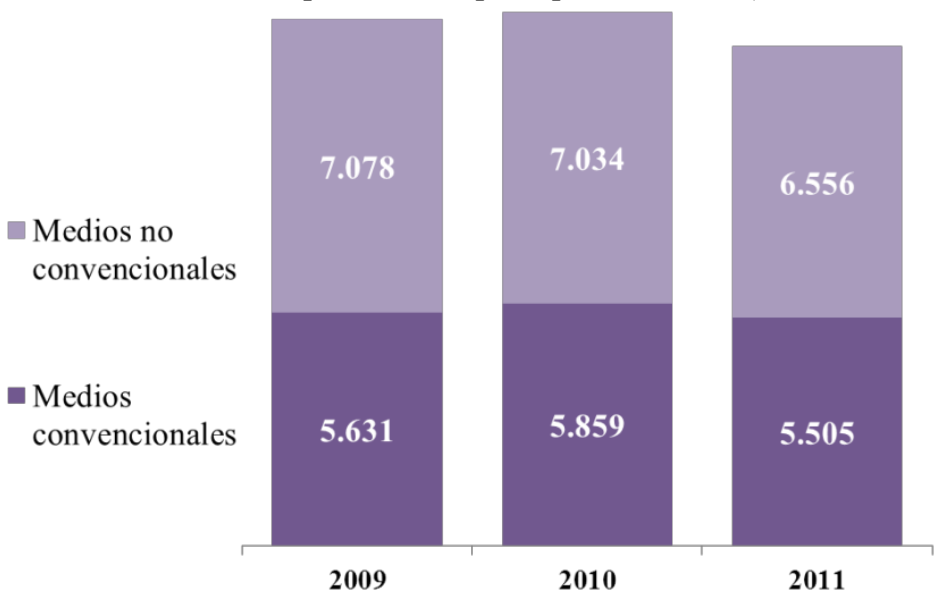

Fuente: InfoAdex

Sin embargo, en valores absolutos, la tendencia es que los medios no convencionales superan a los medios convencionales en cuanto a inversión publicitaria y en parte es gracias a la crisis que obliga a utilizar medios más baratos (que no siempre más rentables si los medimos en términos de coste por impacto) y también 
gracias al cambio de posicionamiento de los primeros: como destaca Charo Sádaba, de la Universidad de Navarra, «es evidente que los medios convencionales se han caracterizado por su mayor creatividad y una línea de venta suave (la soft-sell americana), mientras que, tradicionalmente, los medios no convencionales han sido vistos como menos creativos y más ligados a la venta dura (hard-sell). Esto está cambiando» ${ }^{13}$.

Este comportamiento heterogéneo también se da entre los medios convencionales. Cabe observar en la siguiente tabla que, exceptuando internet y cine, el resto de medios presentan caídas en sus cifras de negocio.

Fig. 4: Inversión publicitaria por medio (en millones de euros).

\begin{tabular}{|c|c|c|c|c|c|c|}
\hline \multicolumn{2}{|c|}{ Medios convencionales } & 2011 & $\% ' 11 /{ }^{\prime} 10$ & 2010 & 2009 & 2008 \\
\hline \multicolumn{2}{|l|}{ Cine } & 25,8 & 5,8 & 24,4 & 15,4 & 21,0 \\
\hline \multicolumn{2}{|l|}{ Diarios } & 967,0 & $-14,0$ & $1.124,4$ & $1.174,1$ & $1.507,9$ \\
\hline \multicolumn{2}{|l|}{ Dominicales } & 67,1 & $-7,0$ & 72,2 & 68,9 & 103,9 \\
\hline \multicolumn{2}{|l|}{ Exterior } & 402,8 & $-4,3$ & 420,8 & 401,4 & 518,3 \\
\hline \multirow[t]{3}{*}{ Internet } & Enlaces patrocinados & 459,9 & 10,2 & 417,2 & 356,4 & 324,4 \\
\hline & Formatos gráficos & 439,3 & 15,1 & 381,7 & 297,7 & 285,6 \\
\hline & Total internet & 899,2 & 12,6 & 798,8 & 654,1 & 610,0 \\
\hline \multicolumn{2}{|l|}{ Radio } & 524,9 & $-4,3$ & 548,5 & $\mathbf{5 3 7 , 3}$ & 641,9 \\
\hline \multicolumn{2}{|l|}{ Revistas } & 381,1 & $-4,2$ & 397,8 & 401,9 & 617,3 \\
\hline \multirow[t]{6}{*}{ Televisión } & Canales de pago & 60,2 & $-7,4$ & 65,0 & 50,0 & 56,1 \\
\hline & TV. autonómicas & 198,0 & $-27,4$ & 272,6 & 237,7 & 319,6 \\
\hline & TV. locales & 2,0 & $-63,6$ & 5,5 & 9,1 & 37,9 \\
\hline & TV. nacionales en abierto & 1977,0 & $-7,1$ & $2.128,8$ & $2.081,1$ & $2.668,8$ \\
\hline & Total televisión & 2237,2 & $-9,5$ & $2.471,9$ & $2.377,8$ & $3.082,4$ \\
\hline & Total & $5.505,1$ & $-6,0$ & $5.858,8$ & $5.630,9$ & $7.102,8$ \\
\hline
\end{tabular}

Fuente: InfoAdex

Aunque la televisión continúa siendo el primer medio por volumen con una participación del 40,6\% del conjunto de medios convencionales, ha perdido un $9.5 \%$, lo que equivale a más de 230 millones de euros respecto al $2010^{14}$. Internet crece a buen ritmo mientras que en el polo opuesto, los diarios están sufriendo duramente los efectos de la crisis con una caída del $14 \%$ respecto al año anterior, alcanzando

13 SÁDABA, C. (2005): «Los medios publicitarios» en Victoria Mas J.S. (coord.), Reestructuras del sistema publicitario, Barcelona, Ariel.

14 El comportamiento de los epígrafes que componen el medio ha sido distinto: los canales nacionales en abierto han caído el $7.1 \%$ (mientras que en 2010 crecieron el $2.3 \%$ respecto al 2009), las televisiones autonómicas reflejan un decremento del $27.4 \%$ (en 2010 crecieron el $14.7 \%$ ) y las televisiones locales se desploman el $63.6 \%$ (el año pasado también perdieron el $39.5 \%$ en su cifra de negocio). 
en 2011 una inversión publicitaria de 967 millones de euros, cifra que aún les permite seguir ocupando el segundo lugar por volumen de negocio.

Las previsiones para el 2012 tampoco son muy optimistas en un entorno de incertidumbre económica: se prevé que siga la tendencia negativa del PIB y de la demanda nacional; el consumo sufrirá un retroceso arrastrado por la caída del empleo, el deterioro de las expectativas, el elevado precio de la energía y el aumento de los impuestos. Las perspectivas para la inversión publicitaria no son pues alentadoras y no hay indicios para pensar en su recuperación a corto y medio plazo. Se prevé que la inversión publicitaria pueda retroceder un $10,7 \%$, según el estudio «El índice de inversión publicitaria (i2p)» de Arce Media ${ }^{15}$.

\section{Objetivo del estudio: La digitalización como eje de transformación de las agencias de medios españolas}

A lo largo de este artículo vamos a tratar de explorar algunos de los factores principales que condicionan el futuro de las agencias de medios españolas en este contexto de enorme dificultad. Para ello, revisaremos la escasa bibliografía existente, nacional e internacional, tanto desde el ámbito de estudio académico como profesional, y resumiremos las tendencias principales que pueden observarse en el sector.

No fue hasta mediados de los años noventa del pasado siglo cuando la popularización de internet convirtió a ese medio en un nuevo vehículo publicitario ${ }^{16}$. Desde entonces, internet y el proceso de digitalización del resto de medios han revolucionado la publicidad. Como bien refleja Patricia Corredor, la industria publicitaria tendrá que realizar un gran esfuerzo:

El impacto de la crisis y la revolución digital están actuando como importantes catalizadores de profundas transformaciones en la industria publicitaria. La expansión de las redes digitales no solo está conmocionando el panorama mediático y condicionando la búsqueda de nuevos modelos de negocio sino que, como una correa de transmisión, está afectando a toda la cadena de valor, aspecto que implica directamente a la publicidad, tanto en su papel financiador de los viejos y nuevos medios como a la propia esencia de la comunicación publicitaria ${ }^{17}$.

Para Daniel Solana, director general de la agencia interactiva DoubleYou, no es suficiente añadir internet a la lista de los medios convencionales como la televisión, la prensa, exterior o radio, no es un medio más, no se comporta como tal, «es una realidad paralela, es un medio de medios, un metamedio. En internet, o mejor

15 Arce Media es una compañía de investigación de medios que mide la actividad publicitaria en todos los medios convencionales e indica tendencias hacia el futuro.

16 KiM, S. J. y McMillan, S. J. (2008): «Evaluation of Internet Advertising Research», Journal of Advertising, 37 (1), 99-112.

17 Corredor Lanas, P. (2011), op. cit., 1-4. 
dicho dentro de internet, también existe la televisión, la prensa o la radio» ${ }^{18}$. Los periódicos poseen su versión online, se puede escuchar la radio por internet y la televisión se convierte en interactiva; ante este panorama, para Solana, más que un medio más, internet parece llamado a difuminar las barreras entre los medios tradicionales, a transformarlos ${ }^{19}$.

Ante ese panorama, la agencia de medios debe adaptarse. Una agencia de medios selecciona los medios y soportes ${ }^{20}$ más adecuados para que las marcas contacten con sus consumidores. Puede parecer una definición simple pero su evolución ha sido y es compleja, y desde un punto de vista económico se han convertido en un excelente ejemplo de éxito empresarial y más en un entorno de recesión económica que aun se ve magnificado al cohabitar con un mercado de oferta, al menos en las televisiones nacionales generalistas. Es en estas condiciones donde el papel del especialista cobra mayor importancia: debe garantizar un mayor retorno de la inversión o return on investment (ROI), bien consiguiendo unas mejores condiciones económicas de los medios, bien segmentando mejor al público objetivo al que se dirige la campaña.

El papel estelar que las agencias de medios desempeñan se debe no solo al gran volumen de inversión que gestionan, que suele ser la parte más sustancial del presupuesto de las campañas publicitarias, sino también a la evolución en el entorno de la comunicación donde las marcas, el consumidor y los medios son cada vez más complejos. En este sentido, Francisco Javier Pérez-Latre, de la Universidad de Navarra, subraya el papel del especialista, ya que «permite prestar mayor atención a problemas específicos que los medios plantean, y por otra parte ofrece a los anunciantes un equipo especializado que se dedica fundamentalmente a la tarea publicitaria de medios» ${ }^{21}$.

La compra, la investigación y la consecuente planificación desempeñan aquí un rol crítico. En esta línea, la labor primordial consiste en identificar grupos homogéneos de consumidores con el fin de alcanzar una comunicación one-to-one. Esta segmentación resulta básica en un panorama donde los consumidores presentan necesidades distintas y los medios acentúan esta fragmentación, especialmente, con la entrada de la televisión digital ${ }^{22}$. Si nos centramos en la televisión, la TDT, cuyo proceso de implantación

\footnotetext{
18 Solana, D. (2005a): «Anuncios en internet. La nueva publicidad», Telos (64).

19 SolanA, D. (2005b): «La nueva agencia está por construir» en Victoria Mas J.S. (coord.), Reestructuras del sistema publicitario, Barcelona, Ariel.

${ }^{20}$ Los medios publicitarios son los canales por los que un mensaje publicitario llega al público objetivo, pero debemos distinguir entre medios y soportes publicitarios. Para Carrero y González Lobo, el medio es «el resultado del desarrollo de una técnica en lo que tiene de vehículo para la inserción de mensajes publicitarios», mientras que un soporte es «cada una de las realidades en las que materializa el medio» (Carrero y González Lobo, 2008). Por ejemplo, en el medio prensa los soportes son las diferentes publicaciones; los soportes en radio son las distintas emisoras como la SER, Cope, Onda Cero, 40 Principales, entre otras.

21 PÉrez-Latre, F. J. (1995): Centrales de compra de medios, Navarra, Ediciones Universidad de Navarra (EUNSA).

22 LeKAKOS, G. (2009): «It's personal: Extracting lifestyle indicators in digital television advertising», Journal of Advertising Research, 404-418.
} 
culminó el 4 de abril de 2010, se ha utilizado en España para multiplicar la oferta. Este hecho implica pasar de los seis canales estatales en abierto a los 32 que están operando, sin contar los operadores autonómicos y locales ${ }^{23}$.

Pero no solo la televisión ha sufrido y está sufriendo una notable transformación. En el resto de medios también se están dando cambios importantes: nuevos soportes, nuevos formatos y especialmente encontramos el punto de inflexión con la aparición de internet. El investigador Josep Adolf Estrader lo resume de esta forma:

Puede parecer un tópico, pero soy de los que tienen claro que las empresas mediáticas contemporáneas tienen un antes y un después, a partir de la irrupción de internet. La red ha implicado la creación de medios de comunicación online o electrónicos, un nuevo campo de actuación para la publicidad, una nueva fuente de información para los que crean, elaboran o difunden los contenidos de los medios informativos, un nuevo vehículo de transmisión de contenidos dentro del proceso de producción y un escenario de derrumbe de múltiples iniciativas empresariales surgidas de medios de comunicación y basadas hipotéticamente en el negocio generado por la venta y suscripción de contenidos y productos, así como en la contratación de publicidad para ser exhibida en la misma red. Y podríamos continuar.

Todo eso que explico no es nuevo para nadie. No estoy descubriendo nada. Pero fundamenta la teoría que internet ha marcado un antes y un después en las empresas mediáticas. A éstas, la red les ha ido muy bien tecnológicamente pero les ha supuesto un dolor de cabeza y un problema importante como negocio. Y más de una redefinición, en todos sentidos ${ }^{24}$.

Internet ha sacudido a las empresas de comunicación en su sentido más amplio: desde los propios medios, las redacciones periodísticas, las productoras de contenidos audiovisuales, las agencias de comunicación y publicidad, los fabricantes de bienes de consumo con la llegada de la próxima generación de terminales móviles o la televisión interactiva o los electrodomésticos inteligentes. La aparición de internet es mucho más que la aparición de un nuevo medio como lo fue la televisión, internet pone en contacto de manera directa a anunciantes y consumidores y está afectando ya a todos los medios de comunicación y en consecuencia a las agencias de comunicación. Un claro ejemplo es la aparición los últimos años de un gran número de empresas de comunicación ligadas al medio internet ${ }^{25}$. No hay que olvidar, tal y como opina Martin Sorrell, consejero

23 CASERO RIPOLLÉS, A. (2009): «La implantación de la TDT en España. Transformaciones en la publicidad televisiva», Telos, 79.

24 Estrader, J. A. (2003): «El sector audiovisual després d'internet», Trípodos, 14, 89-96.

25 En España existen diferentes asociaciones relacionadas con las agencias interactivas. Por un lado, IAB Spain (Interactive Advertising Bureau) que se define como la asociación que representa al sector de la publicidad y la comunicación digital. Con 150 empresas asociadas en la actualidad representa al 95\% del sector en nuestro país. Por otro lado, la Asociación de Agencias Digitales agrupa 40 agencias de marketing y comunicación en las que el mundo digital se encuentra en el centro de su organización y servicios. Por último, la Mobile Marketing Association Spain tiene como objetivo principal potenciar al máximo el móvil como herramienta de marketing y cuenta con más de 67 empresas asociadas. 
delegado del grupo británico de comunicación $\mathrm{WPP}^{26}$, que los consumidores pasan entre el $20 \%$ y el $30 \%$ del día conectados a internet y muchos ciudadanos ya acceden a internet desde sus móviles en lugar de hacerlo desde sus ordenadores, en especial gracias al éxito que han tenido el iPhone de Apple, los nuevos smartphone y los teléfonos con Android de Google ${ }^{27}$.

\section{Internet, el medio que más crece}

Desde comienzos de 1996, año en el que el «Estudio general de medios» está midiendo regularmente el uso de internet en España, internet refleja un continuo crecimiento, no solo en facturación, sino en audiencia. Como muestra la siguiente tabla, internet cerró 2011 con más de 16,5 millones de usuarios en el día de ayer, un $6 \%$ más que en el año anterior, y llega ya al $42.5 \%$ de los españoles.

Fig. 5: Evolución de usuarios de internet desde el año 1996 (en porcentaje).

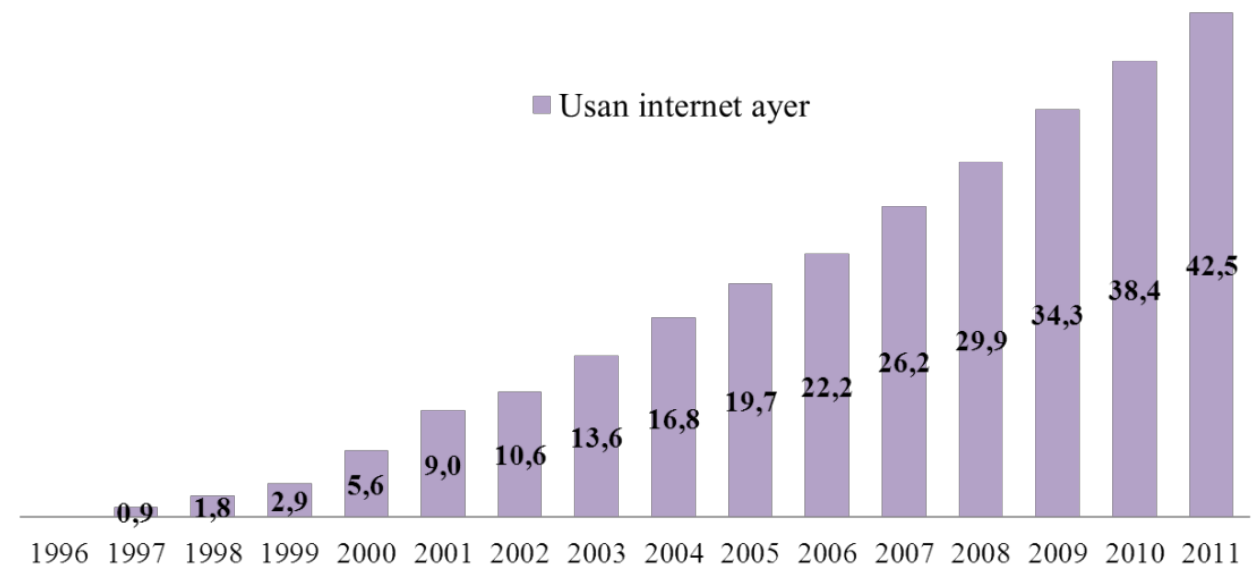

Fuente: AIMC (Asociación para la investigación de medios de comunicación)

26 La corporación británica WPP es uno de los mayores grupos de servicios de comunicación mundiales, junto con Omnicom, Interpublic, Publicis, Dentsu y Havas. Presidido por Martin Sorrell, las agencias de publicidad que forman parte del grupo son Grey, Ogilvy \& Mather, Young \& Rubicam y J. Walter Thompson (JWT), entre otras. Con respecto a las agencias de relaciones públicas destacan Hill \& Knowlton, Ogilvy Public Relations, Burson-Marsteller y Cohn \& Wolfe. También cuenta con institutos de investigación como Kantar Media (que compró recientemente a TNSofres, convirtiéndose así en la principal fuente de información sobre audiencias de televisión en España), BMRB y Millward Brown. También es propietario de la compañía GroupM que agrupa el volumen de las agencias de medios Mindshare, Mediacom, Mediaedge:cia y Maxus.

27 Sorrell, M. (2010): «Los nuevos motores del crecimiento», El Mundo, Madrid, 28 de enero, 6, Extra Publicidad. 
Esta evolución positiva se debe, según algunos autores, a tres motivos principales: la generalización del acceso a través de banda ancha, la mejora y enriquecimiento constante de los contenidos y la creciente adopción de teléfonos móviles de tercera generación y dispositivos portátiles ${ }^{28}$. Según el estudio «Tendencias en móvil en 2012» de Comscore ${ }^{29}$, los dispositivos móviles como los smartphones y las tablets ya superan el $50 \%$ en penetración en España, lo que ha favorecido en particular el crecimiento del uso cotidiano de las redes sociales (el $68,4 \%$ de los internautas accedió ayer y el 79,5\% en la última semana según la última edición del estudio «Navegantes en la red» de $\mathrm{AIMC}^{30}$ ). Las razones no varían mucho de otros informes, las relaciones personales, las aficiones o el trabajo son las más populares, destacando Facebook como la red social más utilizada, tal y como refleja la tabla 6, con más de 16 millones de usuarios registrados en España aunque son Google+, LinkedIn y Twitter las redes con mayor potencial de crecimiento ${ }^{31}$. Esto significa que el poder prescriptor del consumidor online va en aumento y se facilita que el boca-oreja sea más determinante. Otras tendencias como el ascenso del televisor o de las videoconsolas para conectarse a la red también se confirman en este estudio.

Se trata de un medio sin fronteras, que permite poner en contacto a las marcas con los consumidores de un modo inmediato, personal y bidireccional. Y a su vez, los usuarios pueden interactuar entre ellos, creando y compartiendo contenidos informativos. Mientras anunciantes y agencias están haciendo grandes avances para crear campañas utilizando display, search, mobile, streaming video, gaming, social media, gadgets ${ }^{32}$, no debemos olvidar que el reto llega ahora y consiste en ofrecer valor añadido a los consumidores en lugar de simples impresiones ${ }^{33}$. Miguel Ángel

28 Truong, Y., McColl, R. y Kitchen, P. (2010): «Practitioners' perceptions of advertising strategies for digital media», International Journal of Advertising, 29 (5), 709-725.

29 Comscore es una compañía líder en la medición digital y entre los estudios que publica destaca «Tendencias en móvil en 2012». Este informe anual examina el panorama de los dispositivos móviles mediante un estudio sobre las tendencias clave que impulsan el crecimiento de smartphones, el uso de los móviles en redes sociales y comercio electrónico, las dinámicas del sistema de plataformas y los cambios en el consumo de medios digitales.

30 «Navegantes en la red», promovido por AIMC, es uno de los estudios más representativos para conocer el perfil del internauta español así como sus hábitos y opiniones sobre internet.

31 IAB (2011): «III Estudio sobre redes sociales en internet», Madrid.

32 Según la $I A B$, se conoce como publicidad display a la publicidad de formatos gráficos, por contraposición a search o publicidad de enlaces patrocinados por palabras clave. Mobile se define como la publicidad en páginas web y/o wap para móviles. Streaming video consiste en audio y vídeo comprimido que se baja y reproduce a la vez, de este modo el usuario no tiene que esperar a que el archivo se descargue por completo para poder reproducirlo. Gaming o advergaming es la práctica de usar videojuegos para publicitar una marca, girando la trama o dinámica del juego alrededor de ésta. Social media se refiere a la creación de contenidos y aplicaciones por marcas o usuarios que pueden ser compartidos online. Por último, el término gadget se da a una nueva categoría de mini aplicaciones, diseñadas para proveer de información o mejorar una aplicación o servicios de un ordenador, o bien cualquier tipo de interacción a través de internet.

33 KIM, S. J. (2008): «A framework for advertising in the digital age», Journal of Advertising Research, 310-312. 
Pérez, de la Universidad Complutense de Madrid, defiende esta idea cuando afirma que los medios afectados por la revolución tecnológica digital ya están ejerciendo una gran influencia sobre la metodología y la filosofía de trabajo alrededor de la comunicación publicitaria ${ }^{34}$.

Fig. 6: Red social que utiliza en los últimos 30 días (en porcentaje).

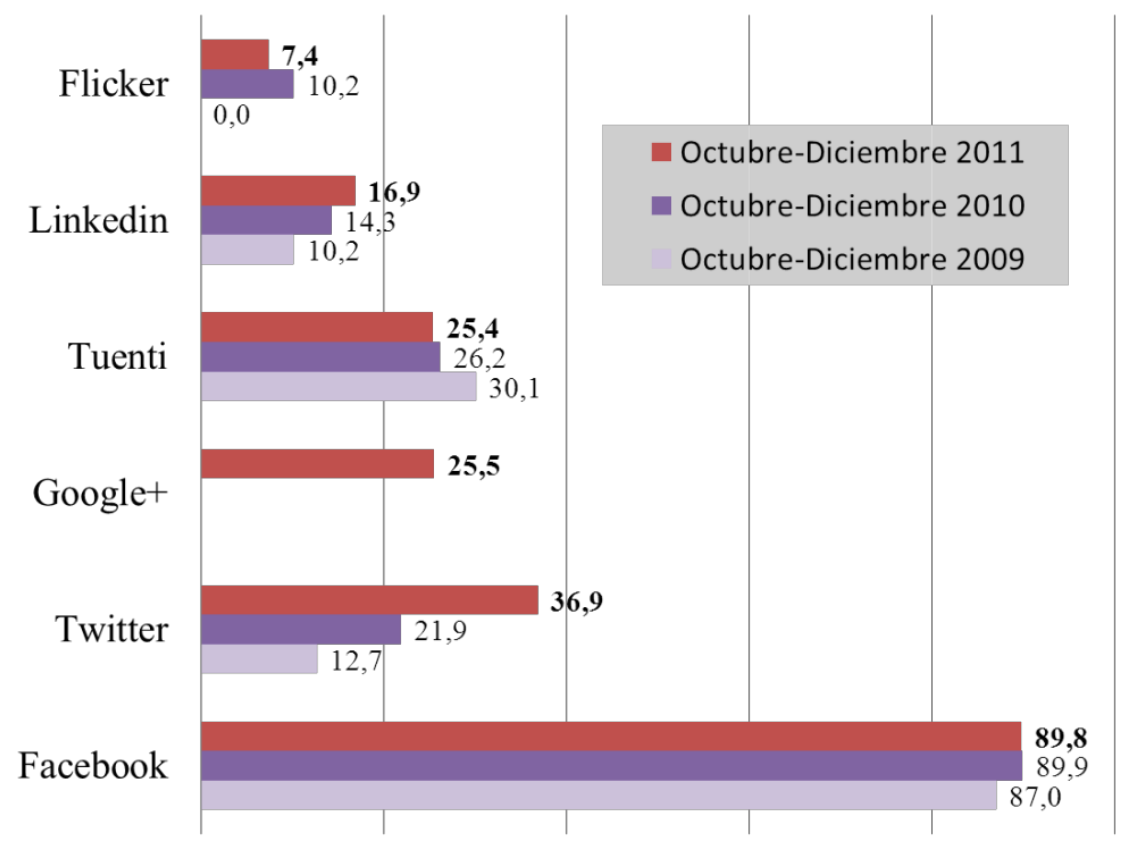

Fuente: AIMC (Asociación para la investigación de medios de comunicación)

Es cierto, como hemos visto, la agencia evoluciona a la par de los medios y constantemente está reinventando nuevos formatos de la mano de la tecnología (publicidad interactiva, microsites, formatos rich media, spots interactivos, advergames, aplicaciones para móviles, anuncios virales ${ }^{35}$ ), pero en este punto queremos

34 PÉRez Ruiz, M. Á. (2005): «Las relaciones entre anunciantes, agencias de publicidad y agencias de medios»» en Victoria Mas J.S. (coord.), Reestructuras del sistema publicitario, Barcelona, Ariel.

35 La publicidad viral es un formato que consiste en que los propios usuarios difunden a través de su ordenador o teléfono móvil un mensaje publicitario y que, según Mònika Jiménez, de la Universidad Pompeu Fabra, está desbancando en efectividad a las formas de publicidad convencionales (Jiménez Morales, 2008). La autora expone algunos ejemplos de casos exitosos de publicidad viral como la campaña de la cadena de televisión MTV "Amo a Laura" (http://www.youtube.com/ watch?v=hRdVg_JATII) y la acción "Levántate ZP" (http://www.youtube.com/watch?v= Nd1q_JIR_B8\&has_verified=1). 
plasmar las reflexiones del profesor Joseba Andoni Etxebarria de la Universidad del País Vasco cuando afirma que «el origen más profundo de los cambios debemos buscarlo en la evolución de los anunciantes y de sus necesidades comunicativas ${ }^{36}$. Para Stephen J. Kim, de Microsoft ${ }^{37}$, los consumidores esperan de los medios, en particular de los medios digitales, entretenimiento y experiencias comunicativas. Añade que el $84 \%$ de los internautas de 14 a 24 años rechazan la publicidad que «interrumpe y repite; sin embargo, aceptan formas de publicidad que sean relevantes para sus necesidades, a la medida de sus comunidades y que les ofrezca valor ${ }^{38}$. Las estrategias publicitarias en el entorno digital deben replantearse. Algunos autores se atreven ya a proponer algunos principios generales; entre ellos se encontraría, por ejemplo, la sensibilidad hacia la privacidad y las molestias de los mensajes no deseados, la confianza en el emisor ${ }^{39}$, la persuasión adaptada de forma natural a la esfera social, la utilización de la participación voluntaria de los usuarios o el uso de sistemas de geolocalización ${ }^{40}$.

Como se ha expuesto, internet es el único medio que experimenta crecimientos positivos ininterrumpidamente en su inversión. Podríamos ahora plantear la hipótesis de que internet, al ser el medio que más crece en cuanto a inversión publicitaria (en concreto el $12,6 \%$ respecto al año anterior), es el medio que gana más cuota de inversión respecto a otros medios, esto es, que se desvía inversión del resto de medios hacia internet. La siguiente tabla lo confirma:

Se observa cómo el 2009 fue el primer año en el que la televisión experimentó una bajada e internet se posicionó por primera vez como la tercera opción en cuota, posición que se consolidó en 2010 al alcanzar el 13,5\%. En 2011 se ha acercado al segundo lugar que históricamente han ocupado los diarios y podemos prever que en el 2012, internet se convertirá en el segundo medio en términos de inversión publicitaria. Se ratifica, de esta manera, cómo la distribución de la inversión publicitaria está cambiando de dirección hacia internet, rompiendo con la tendencia reflejada en los planes de medios más tradicionales, en los que la televisión era el medio principal e incuestionable. En nuestra opinión, se trata del gran cambio de los últimos años.

36 EtXebarRia, J. A. (2005): «Las empresas publicitarias» en Victoria Mas J.S. (coord.), Reestructuras del sistema publicitario, Barcelona, Ariel, 101-139.

37 Stephen Kim es director del Global Branded Entertainment and Experiences Team for Microsoft Digital Advertising Solutions. Está especializado en estudiar los efectos de la publicidad digital, la medición del ROI en la publicidad, social media marketing, la eficacia del streaming video y el in-game marketing.

38 KIM, S. J. (2008), op. cit., 310-312.

39 TAYLOR, C. R. (2009): «The Six Principles of Digital Advertising», International Journal of Advertising, 28 (3), 411-418.

40 DJAvit, N. (2010): «10 trends in digital marketing», AdMap, Marzo, 14-15. 
Fig. 7: Cuota de la inversión publicitaria por medio (en porcentaje).

\begin{tabular}{|l|c|c|c|c|c|}
\hline & 2007 & 2008 & 2009 & 2010 & 2011 \\
\hline Televisión & $43,4 \%$ & $43,4 \%$ & $42,1 \%$ & $42,3 \%$ & $40,6 \%$ \\
\hline Diarios & $23,7 \%$ & $21,2 \%$ & $20,9 \%$ & $19,2 \%$ & $17,6 \%$ \\
\hline Radio & $8,5 \%$ & $9 \%$ & $9,5 \%$ & $9,4 \%$ & $9,5 \%$ \\
\hline Exterior & $7,1 \%$ & $7,3 \%$ & $7,1 \%$ & $7,2 \%$ & $7,3 \%$ \\
\hline Revistas & $9 \%$ & $8,7 \%$ & $7,1 \%$ & $6,8 \%$ & $6,9 \%$ \\
\hline Internet & $6 \%$ & $8,6 \%$ & $11,6 \%$ & $13,5 \%$ & $16,3 \%$ \\
\hline Dominical & $1,7 \%$ & $1,5 \%$ & $1,2 \%$ & $1,2 \%$ & $1,2 \%$ \\
\hline Cine & $0,5 \%$ & $0,3 \%$ & $0,3 \%$ & $0,4 \%$ & $0,5 \%$ \\
\hline TOTAL & $100 \%$ & $100 \%$ & $100 \%$ & $100 \%$ & $100 \%$ \\
\hline
\end{tabular}

Fuente: Estudio InfoAdex

\section{La medición de internet}

Desde el punto de vista de la planificación, son muchas las ventajas que presenta este medio: la posibilidad de segmentación y de alcanzar a públicos objetivos concretos; su flexibilidad en cuanto a los formatos publicitarios que puede admitir y que a su vez están en permanente actualización ${ }^{41}$ gracias, una vez más, a la tecnología; también la tecnología permite que puedan personalizarse los mensajes publicitarios que recibe un determinado usuario; el fenómeno 2.0 permite la interactividad entre marcas y consumidores y los contenidos son generados y compartidos por los propios usuarios; y por supuesto el hecho de que permite medir los resultados con gran detalle, podríamos decir que podemos conocer de un modo preciso lo que llamamos la trazabilidad o seguimiento de un click. Sin embargo, la auditoría de audiencias actual no ha seguido el ritmo con el que ha avanzado la tecnología y el nuevo comportamiento de los consumidores ${ }^{42}$. Chun-Yao Huang, de la London Business School, también está de acuerdo con lo anterior cuando expone que se abre una gran oportunidad para medir el comportamiento de la audiencia ${ }^{43}$.

41 Surgen como hemos visto alternativas a los formatos más clásicos como el display: Programas de afiliación, patrocinios online, cupones que se han convertido junto con el search-engine marketing $(S E M)$ en herramientas para crear no solo imagen de marca sino que eventualmente pueden desembocar en compras (Pfeiffer\&Zinnbauer, 2010).

42 Wurtzel, A. (2009): «Now. Or never: An urgent call to action for consensus on new media metrics», Journal of Advertising Research, 263-265.

43 HuAnG, C. Y. y Lin, C. S. (2006): «Modeling the audience's banner ad exposure for internet advertising planning», Journal of Advertising, 35 (2), 123-136. 
En primer lugar, nos preguntamos: ¿Cuál es el sistema que hoy predomina en la medición de la audiencia en internet? Hoy en día, cohabita en los planes digitales un doble sistema de medición, por un lado, el de la cobertura en términos de usuarios únicos y número de impresiones, por otro lado, los resultados en términos de click-through rate (CTR); en otras palabras, se da una dicotomía en los objetivos de marketing en internet: respuesta directa versus branding ${ }^{44}$.

Investigaciones recientes han demostrado que evaluando únicamente el clickthrough rate, claramente indicando un coste por conversión o por resultados, se tiene solo una imagen parcial de la eficacia en internet. No es suficiente, pues, acudir al ratio de conversión $C T R$ para medir el éxito de una campaña online: habrá que analizar en profundidad si la visita reacciona ante el producto tan bien como ha reaccionado ante la publicidad, esto es, si contrata los servicios, navega por la web de información, se suscribe a algún servicio informativo o compra algún producto en dicha web. En la misma línea se manifiesta León Saez de Ybarra, de la Universidad del País Vasco, quien afirma que «considerar la eficacia solo desde el punto de vista de los clicks induciría una impresión errónea, pues lo relevante es ver en qué medida influyen los clicks sobre la base de visitantes de las webs del anunciante, al cual en último término remiten los banners» ${ }^{45}$.

\section{Conclusiones}

\subsection{La individualización del consumo de medios}

Como se ha ido plasmando durante el desarrollo de este artículo, el presente y el futuro de todos los actores (agencias, anunciantes, medios) está en revisión y lleno de interrogantes. La tecnología aparece como un factor clave tanto para el desarrollo de nuevos medios de comunicación como de nuevas posibilidades creativas. En este sentido, la revolución de los medios ya está aquí: internet introduce el factor de la personalización en el consumo audiovisual, que el usuario demandará inexcusablemente con independencia de la plataforma de distribución ${ }^{46}$. Ya han llegado los nuevos televisores con acceso directo a internet y las tarifas planas de acceso a internet desde los teléfonos móviles permiten que la web se meta en nuestros bolsillos. Una revolución tecnológica que posibilita que el consumidor pueda acceder a contenidos audiovisuales en movilidad y a través de tres pantallas principales (la

\footnotetext{
44 BRoussaRD, G. (2000): «How advertising frequency can work to build online advertising effectiveness», International Journal of Market Research, 42 (4), 439-457.

45 LEÓN SAEZ DE YBARRA, J.L. (2009): «Nuevos soportes y mercados de la publicidad digital. Transiciones y experiencias», Pensar la Publicidad. Revista internacional de investigaciones publicitarias, 3 (2), 17-30.

46 García García, A. y Vinader Segura, R. (2010): «Televisión tradicional y televisión móvil. Estrategias para contenidos televisivos en movilidad», Telos, 83.
} 
del televisor, la del ordenador y la del teléfono móvil ${ }^{47}$, aunque a menudo se incluye una cuarta pantalla, la consola portátil de videojuegos).

Es evidente que el acceso e intercambio de información se dará, cada vez más, a través de los dispositivos portátiles como los smartphones, netbooks y e-books ${ }^{48}$. El móvil cada vez tiene más importancia para los anunciantes, por un lado, la audiencia de internet desde los dispositivos móviles no para de crecer, por otro, también permite una alta segmentación de la audiencia por variables sociodemográficas e incluso por geolocalización. Ya no es extraño que mediante el GPS o la triangulación se pueda detectar la localización de un individuo que está, por ejemplo, buscando un restaurante italiano, y con un simple click el terminal móvil nos facilite el nombre, la dirección y el teléfono de los tres restaurantes italianos más próximos ${ }^{49}$. La actividad publicitaria lo corrobora ya que las previsiones de inversión en marketing móvil en España son optimistas con un crecimiento medio del $62 \%$ para el 2012 y 2013, año en que se espera superar los 160 millones de euros ${ }^{50}$.

Del estudio «Uso de internet desde el móvil: tendencias de futuro» realizado por Nielsen Online, compañía especializada en la medición de audiencias en internet, y TAPTAP Networks, red especialista en publicidad móvil, pueden destacarse los siguientes datos ${ }^{51}$ :

- La audiencia de internet desde dispositivos móviles se ha incrementado en un $47 \%$.

- Principalmente se utiliza para buscar información en portales y buscadores y acceder al correo electrónico.

- Aumenta especialmente el uso de las redes sociales.

- En cuanto a su uso publicitario: de momento el $89 \%$ de las campañas publicitarias de 2009 se centraron en el diseño de banners.

- En el futuro, las marcas usarán formatos más innovadores que aprovecharán la posibilidad que ofrecen los móviles de realizar acciones como el click to call (solicitud de una llamada comercial) o el click to app (lanzamiento de una aplicación).

47 No existe en la actualidad una cuota de mercado lo suficientemente representativa para considerar a la televisión en el móvil como un sistema de distribución de contenidos con garantías de éxito. Sin embargo, si analizamos el móvil como dispositivo de comunicación las cosas cambian: sigue siendo el mercado con más tirón de las telecomunicaciones y el número de abonados sigue aumentando en todas las regiones del mundo, incluyendo los países con economías emergentes (García García \& Vinader Segura, 2010).

48 Cerezo, J. M. (2010): «Smartphone. Toda la información al alcance de tu mano», Telos, 83.

49 O’Reilly, T., \& BAtTelle, J. (2009): «Web Squared: Web 2.0 Five Years On», Web 2.0 Conference

50 Mobile Marketing Association (2012): «4 Estudio de inversión en publicidad y marketing móvil en España 2011», Madrid.

51 TAPTAP NETWORKS \& THE NiELSEN COMPANY (2010): «Uso de internet desde el móvil: tendencias de futuro», Madrid. 


\subsection{Hacia un nuevo modelo en el ecosistema audiovisual actual}

Se abren pues nuevas posibilidades en el momento de planificar una campaña publicitaria ante un entorno televisivo revolucionado por sustanciales cambios legislativos, importantes cambios empresariales, un creciente nivel de fragmentación de audiencia, la proliferación de nuevos consumos y las consecuencias de la desaparición de la publicidad en TVE. En este sentido, Patricia Corredor lo resume afirmando que «en la actualidad, la multiplicación exponencial de los canales y soportes, la fragmentación de las audiencias y los nuevos usos sociales de los usuarios imponen nuevas reglas de juego a la publicidad basadas en la interactividad $»^{52}$. Como señalan Fernández Cavia y Puigpinós: «Un nuevo consumidor y un nuevo panorama de medios de comunicación exigen una nueva agencia de publicidad y, por consiguiente, un nuevo profesional publicitario» ${ }^{53}$, y predicen que aquellas empresas y profesionales que no se renueven van encaminados a desaparecer, siendo remplazados por nuevas empresas más pequeñas, flexibles y especializadas.

Ante este nuevo ecosistema comunicativo, las agencias de medios están respondiendo con un reposicionamiento de su papel como empresas. Por un lado, las grandes agencias tienden a expandir su catálogo de servicios, que con mayor frecuencia incluye servicios de estrategia y creatividad. Lejos de limitarse a su función tradicional de centrales de compra de espacio y tiempo en los medios de comunicación, proponen a los anunciantes nuevas estrategias y nuevos formatos para relacionar a las marcas con los consumidores. En ese sentido, pueden verse como una creciente competencia para las agencias de servicios plenos o para las agencias creativas. Por otro lado, se presentan ante las empresas como proveedores de investigación, no sólo de audiencias, sino también de comportamiento del consumidor y de la eficacia de la publicidad. De esta manera, disputan a la agencia publicitaria su papel como consultora de comunicación del anunciante.

Cualquier crisis es no sólo un tiempo de dificultades, sino también de oportunidades. Y las grandes agencias de medios cuentan con la experiencia, el conocimiento y la capacidad de inversión necesarias para fortalecer su posición dentro de la industria publicitaria en esta época de cambios.

\subsection{El gran reto se encuentra en la medición de la audiencia en internet}

Lo hemos intentado describir en el artículo: la inversión publicitaria global sigue descendiendo, nos encontramos ante una mayor complejidad en el panorama de los medios y se consolidan las inversiones publicitarias online. Sin embargo, en 2010 se puso de manifiesto que el medio internet no estaba preparado para asumir las inversiones que buscaban alternativas a la televisión desde el momento en que ésta inflacionó sus costes publicitarios entre un $25 \%$ y un $40 \%$ como consecuencia de la reducción de la

52 Corredor Lanas, P. (2011), op. cit., 1-4.

53 Fernández Cavia, J. y PuigPinós, A. (2010): «Crisis and change in the advertising industry», IAMCR Conference, Braga, Portugal. 
oferta del espacio publicitario desde la salida de la publicidad de TVE. Según Jon Artolozaga, director de investigación en la agencia de medios Mindshare, se preveía que internet iba a recibir mucho dinero procedente de la televisión huyendo del incremento de precios. Sin embargo, este efecto no se produjo en toda su intensidad debido a que el medio online no tiene aún la infraestructura necesaria para gestionar campañas masivas, en parte, porque existe excesiva fragmentación y porque no cuenta con métricas comparables al resto de medios en términos de cobertura, OTS y $G R P^{54}$, lo que dificulta los trasvases de inversión que hasta ahora se miden de una forma muy determinada y esperan los mismos estándares en el nuevo medio.

Aún queda mucho trabajo por hacer en términos de entender cómo es la mejor manera de recopilar información sobre los usuarios, cómo determinar y medir los niveles ideales de interactividad, la personalización y la interacción social de audiencias concretas, y cómo desarrollar mejores indicadores de éxito para medir las acciones online a largo plazo ${ }^{55}$.

Ante esta apuesta clara por internet, las agencias de medios han empezado a desarrollar nuevos indicadores de medición que permiten justificar las inversiones online con los mismos argumentos que se utilizan con el resto de medios: es el caso del $i G R P$, esto es, el GRP en internet, una nueva métrica que está remplazando ya a los indicadores más convencionales como impresiones y clicks y que permite comparar por primera vez los objetivos y resultados en internet con los del resto de medios ${ }^{56}$.

Una de las consecuencias ha sido que las acciones de internet ya pueden integrarse plenamente en un plan de medios, no es ya un nuevo medio, se ha convertido en un medio más que puede analizarse con las mismas métricas que el resto de medios, ganando día tras día cuota de inversión publicitaria y aspirando a convertirse en el segundo medio en cuanto a inversión publicitaria, lugar que hoy ocupa la prensa con el 17,6\% del share of spending.

Como consideran Carmen López y Emma Torres, de la Universidad de Vigo, «el futuro del éxito publicitario pasa por la capacidad que muestren las marcas de sorprender, pero sorprender en sentido amplio y no sólo con mensajes transgresores, provocativos o alternativos. Hacen falta medios de difusión distintos» ${ }^{57}$. Por lo

\footnotetext{
54 Llamamos cobertura al número de personas del grupo objetivo que resultan impactadas al menos una vez por la campaña, también se puede expresar en porcentaje. OTS proviene del inglés opportunity to see, es decir, el número de veces o frecuencia media con que los individuos del grupo objetivo han entrado en contacto con la campaña. GRP, del inglés gross rating point, representa el número de impactos producidos por una campaña expresado como porcentaje del grupo objetivo. (Carrero y González Lobo, 2008).

55 HILL, R.P. y MORAN, N. (2011), op. cit., 1-4.

56 El $i G R P$ se calcula sobre la base de universos genéricos (aceptados por la industria publicitaria) no solo de internautas (como lo hacen los medidores tradicionales en internet) para de este modo ser comparable con la planificación en el resto de medios.

57 LÓPEZ De Aguileta, C. y TorRes, E. (2007): «Medios y soportes alternativos para una publicidad convencional: publicidad off the line», Pensar la Publicidad. Revista internacional de investigaciones publicitarias, 1 (2), 117-130.
} 
tanto las agencias de medios están obligadas a evolucionar para responder al entorno que hemos descrito.

\section{Referencias bibliográficas}

AIMC (2012a): «Navegantes en la red 2012», Madrid.

- (2012b): «Estudio general de medios», Madrid.

ARCE MEDIA (2012), «El índice de inversión publicitaria (i2p)», Madrid.

BoIX, A. (2007): «Transformaciones en el ecosistema mediático y nuevas pautas de regulación administrativa del hecho audiovisual», Quaderns del CAC, 27, 37-51.

BROUSSARD, G. (2000): «How advertising frequency can work to build online advertising effectiveness», International Journal of Market Research, 42 (4), 439-457.

CARdoso, G. (2011): «Más allá de internet y de los medios de comunicación de masas», Telos, 86, 1-10.

CARrero, E., y GonzÁlez Lobo, M. Á. (2008): Manual de planificación de medios: Todo lo que hay que saber para planificar correctamente los medios, Madrid, ESIC.

CASERO RIPOLLÉS, A. (2009): «La implantación de la TDT en España. Transformaciones en la publicidad televisiva», Telos, 79.

CAstellblanQue, M. (2001): Estructura de la actividad publicitaria. La industria de la publicidad de la A a la Z. España: Un caso extrapolable, Barcelona, Ediciones Paidós.

CEREZO, J. M. (2010): «Smartphone. Toda la información al alcance de tu mano», Telos, 83.

COMSCORE (2012): «Tendencias en móvil en 2012», Madrid.

CORREDOR LANAS, P. (2011): «Nuevos profesionales en publicidad», Telos, 87, 1-4.

DJAVIT, N. (2010): «10 trends in digital marketing», AdMap, Marzo, 14-15

ESTRADER, J. A. (2003): «El sector audiovisual després d'internet», Trípodos, 14, 89-96.

ETXEBARRIA, J. A. (2005): «Las empresas publicitarias» en Victoria Mas J.S. (coord.), Reestructuras del sistema publicitario, Barcelona, Ariel, 101-139.

FERNÁNDEZ CAVIA, J. (2005): «Los formatos no convencionales y la transformación del negocio publicitario en la televisión», Quaderns del CAC, 22, 35-48.

FERnÁNDEZ CAVIA, J. y PUIGPINÓS, A. (2010): «Crisis and change in the advertising industry», IAMCR Conference, Braga, Portugal.

GARCÍA GARCÍA, A. y VinAder SEgURA, R. (2010): «Televisión tradicional y televisión móvil. Estrategias para contenidos televisivos en movilidad», Telos, 83.

HILL, R.P. y MORAN, N. (2011): «Social marketing meets interactive media: lessons for the advertising community», International Journal of Advertising, 30, 5.

HuANG, C. Y. y LIN, C. S. (2006): «Modeling the audience's banner ad exposure for internet advertising planning», Journal of Advertising, 35 (2), 123-136.

HuLL, J. (2009): «Conclusion: the future of advertising and advertising agencies» en $\mathrm{H}$. Powell, H. [et al.] (ed), The advertising handbook, London, Routledge.

IAB SPAIN (2011): «III Estudio sobre redes sociales en internet», Madrid.

- (2012): «Glosario de términos de publicidad y marketing digital», Madrid.

INFOADEX (2012): «Estudio de la inversión publicitaria en España 2012», Madrid.

JIMÉNEZ MORALES, M. (2008): «Publicidad viral: la comunicación por contagio», Hipertext.net, 6 . 
KIM, S. J. (2008): «A framework for advertising in the digital age», Journal of Advertising Research, 310-312.

KIM, S. J. y MCMiLlan, S. J. (2008): «Evaluation of Internet Advertising Research», Journal of Advertising, 37 (1), 99-112.

LEKAKOS, G. (2009): «It's personal: Extracting lifestyle indicators in digital television advertising», Journal of Advertising Research, 404-418.

LEÓN SAEZ DE YBARRA, J.L. (2009): «Nuevos soportes y mercados de la publicidad digital. Transiciones y experiencias», Pensar la Publicidad. Revista internacional de investigaciones publicitarias, 3 (2), 17-30.

LóPez De AguiletA, C. y TorRes, E. (2007): «Medios y soportes alternativos para una publicidad convencional: publicidad off the line», Pensar la Publicidad. Revista internacional de investigaciones publicitarias, 1 (2), 117-130.

MOBILE MARKETING ASSOCIATION (2012): « $44^{\circ}$ Estudio de inversión en publicidad y marketing móvil en España 2011», Madrid.

O’Reilly, T., \& BAtTelle, J. (2009): «Web Squared: Web 2.0 Five Years On», Web 2.0 Conference.

PÉREZ-LATRE, F. J. (1995): Centrales de compra de medios, Navarra, Ediciones Universidad de Navarra (EUNSA).

- (2000): Planificación y gestión de medios publicitarios. Barcelona, Ariel.

PÉREZ RUIZ, M. Á. (2005): «Las relaciones entre anunciantes, agencias de publicidad y agencias de medios» en Victoria Mas J.S. (coord.), Reestructuras del sistema publicitario, Barcelona, Ariel.

PFEIFFER, M. y ZINNBAUER, M. (2010): «Can old media enhance new media?», Journal of Advertising Research, 42-49.

SÁDABA, C. (2005): «Los medios publicitarios» en Victoria Mas J.S. (coord.), Reestructuras del sistema publicitario, Barcelona, Ariel.

SolanA, D. (2005a): «Anuncios en internet. La nueva publicidad», Telos (64).

- (2005b): «La nueva agencia está por construir» en Victoria Mas J.S. (coord.), Reestructuras del sistema publicitario, Barcelona, Ariel.

Sorrell, M. (2010): «Los nuevos motores del crecimiento», El Mundo, Madrid, 28 de enero, 6, Extra Publicidad.

TAPTAP NETWORKS \& THE NIELSEN COMPANY (2010): «Uso de internet desde el móvil: tendencias de futuro», Madrid.

TAYLOR, C. R. (2009): «The Six Principles of Digital Advertising», International Journal of Advertising, 28 (3), 411-418.

TRUONG, Y., MCCOLL, R. y KiTCHEN, P. (2010): «Practitioners' perceptions of advertising strategies for digital media», International Journal of Advertising, 29 (5), 709-725.

Vollmer, C., \& Precourt, G. (2008): Always on. Advertising, marketing and media in an era of consumer control, New York, McGraw Hill.

WuRTZEL, A. (2009): «Now. Or never: An urgent call to action for consensus on new media metrics», Journal of Advertising Research, 263-265.

Recibido: 21 de marzo de 2012

Aceptado: 29 de junio de 2012 